\title{
SPARSITY-BASED MULTI-TARGET LOCALIZATION EXPLOITING MULTI-FREQUENCY COPRIME ARRAY
}

\author{
Si Qin, Yimin D. Zhang, and Moeness G. Amin \\ Center for Advanced Communications, Villanova University, Villanova, PA 19085, USA
}

\begin{abstract}
In this paper, a novel sparsity-based multi-target localization approach is proposed by exploiting a coprime array operated with multiple narrowband signals of distinct but closely separated carrier frequencies. The cross-covariance matrix is formulated between baseband array data corresponding to different sensing frequencies to generate virtual difference coarrays, which enable direction-of-arrival (DOA) estimation of more targets than the number of physical sensors. In addition, the use of well designed multi-frequency signals unwraps the propagation phase information, thereby enabling unambiguous estimation of the target ranges. The DOA and range estimations are cast as a sparse reconstruction problem and are solved using the complex mulititask Bayesian compressive sensing (CMT-BCS) technique. The effectiveness of the proposed technique is verified through simulation results.
\end{abstract}

Index Terms - Target localization, DOA estimation, coprime array, Bayesian compressvie sensing, sparse array

\section{INTRODUCTION}

Target localization is an important area that finds variety of applications in radar, sonar, communication, and navigation [1]-[4]. In recent years, simultaneous localization of multiple targets has been intensively investigated using array processing and multiple-input multiple-output (MIMO) systems (e.g., [5]-[8]). The target location information can be characterized using range, angle, or both [9]-[11]. In this paper, target location is determined using the DOA and range information. The number of detectable targets in DOA estimation is determined by the number of degrees-of-freedom (DOFs) of the array, i.e., an $N$-element uniform linear array (ULA) can localize up to $N-1$ targets by using conventional methods. To increase the number of detectable targets, sparse arrays are often used to achieve a higher number of DOFs through the exploitation of the coarray concept [12]. Among a number of techniques that are available for sparse array construction, coprime array is very attractive due to its systematical design capability and DOF analysis [13]-[15]. In addition, the coprime array configurations can also be implemented using a ULA with signal measurement made in two coprime frequencies [16],[17].

In this paper, we consider target localization problems in a coprime array using a set of continuous-wave $(\mathrm{CW})$ sig-

This work was supported in part by the Office of Naval Research (ONR) under grant N00014-13-1-0061. nals with distinct but closely separated frequencies. Direct search of targets in both range and DOA domains requires computational intensive operations. As we cannot recover signal waveforms of more sources than the number of sensors in either the physical array or difference coarray context, the number of detectable targets may still subject to the number of physical array sensors when performing a direct search.

In the novel technique developed in this paper, the joint DOA and range estimation is cast as a sparse reconstruction problem and solved by exploiting the compressive sensing (CS) techniques [18]. In particular, the cross-covariance matrix obtained from the baseband signals corresponding to different sensing frequency pairs is first formulated to generate difference coarrays. In this way, the number of detectable targets is determined by the number of virtual sensors which is usually much higher than the number of physical sensors. The positions of the nonzero entries of the resulting sparse vector correspond to the signal DOAs in the discretized spatial domain, whereas the phase information of each nonzero entry carries the target range information as it corresponds to the propagation phase difference of the different frequency pair. However, due to phase wrapping, the phase information obtained from a single frequency pair does not yield meaningful range estimation. The use of well designed multiple frequencies allows effective phase unwrapping for unambiguous range estimation.

A high number of CS algorithms have been proposed to solve the joint DOA and range estimation problem. As the preferred approach, we use CS algorithms in the Bayesian sparse learning context as they achieve superior performance and are insensitive to the coherence of dictionary entries. To handle the complex-valued observations and entries in the underlying problem, the complex multitask Bayesian compressive sensing (CMT-BCS) [19] is used in this paper. The CMTBCS achieves improved sparse signal reconstruction because it utilizes the group sparsity of the real and imaginary components as they are merely the projection of the same complex value into two orthogonal axes.

Notations: We use lower-case (upper-case) bold characters to denote vectors (matrices). In particular, $\mathbf{I}_{N}$ denotes the $N \times N$ identity matrix. (.)* implies complex conjugation, whereas $(.)^{T}$ and $(.)^{H}$ respectively denote the transpose and conjugate transpose of a matrix or vector. $\operatorname{vec}(\cdot)$ denotes the vectorization operator that turns a matrix into a vector by stacking all columns on top of the another, and $\operatorname{diag}(\mathbf{x})$ denotes a diagonal matrix that uses the elements of $\mathrm{x}$ as its diagonal elements. $\mathrm{E}(\cdot)$ is the statistical expectation operator and 
$\bigotimes$ denotes the Kronecker product. $P_{r}(\cdot)$ denotes the probability density function (pdf), and $\mathcal{N}(x \mid a, b)$ denotes that random variable $x$ follows a Gaussian distribution with mean $a$ and variance $b . \operatorname{Re}(x)$ and $\operatorname{Im}(x)$ denote the real and imaginary parts of complex element $x$, respectively.

\section{SIGNAL MODEL}

Without loss of generality, we limit our discussion in this paper to far-field targets in the two-dimensional (2-D) space where the DOA is described by the azimuth angle only. Extension to three-dimensional (3-D) space is straightforward.

Consider a scene with $Q$ far-field targets whose location is modeled as $\left(\theta_{q}, R_{q}\right), q=1,2, \cdots, Q$. As shown in Fig. 1, a coprime array utilizes a coprime pair of uniform linear subarrays, i.e., $2 M$-element subarray with an interelement spacing of $N$ units, and $N$-element subarray with an interelement spacing of $M$ units [14]. The unit interelement spacing is denoted as $d$. The integer numbers $M$ and $N$ are chosen to be coprime $(M<N)$, i.e., their greatest common divisor is one. The array sensors are positioned at

$\mathbb{P}=\{n M d \mid 0 \leq n \leq N-1\} \bigcup\{m N d \mid 0 \leq m \leq 2 M-1\}$

Because the two subarrays share the first sensor at the zeroth position, the total number of the sensors used in the coprime array is $2 M+N-1$. Denote $\mathbf{p}=\left[p_{1}, \ldots, p_{2 M+N-1}\right]^{T}$ as the positions of the array sensors where $p_{i} \in \mathbb{P}, i=1, \ldots, 2 M+$ $N-1$. The first sensor, located at $p_{1}=0$, is assumed as the reference.

Assume $K$ continuous-wave (CW) signals are emitted from an antenna collocated with the coprime receive array. For a CW waveform with frequency $f_{k}, k=1, \ldots, K$, the signal received at the coprime array is expressed as

$$
\check{\mathbf{x}}_{k}(t)=\exp \left(j 2 \pi f_{k} t\right) \sum_{q=1}^{Q} \rho_{q}(t) e^{-j \frac{4 \pi f_{k}}{c} R_{q}} \mathbf{a}_{k}\left(\theta_{q}\right)+\check{\mathbf{n}}_{k}(t),
$$

where $\rho_{q}(t), q=1, \ldots, Q$, are complex scattering coefficients of the targets, which are assumed to be uncorrelated zero-mean random variables with $E\left[\rho_{q}^{*} \rho_{p}\right]=\sigma_{q}^{2} \delta_{q, p}, 1 \leq$ $q, p \leq Q$, due to, e.g, the radar cross section (RCS) fluctuations. In addition, $c$ is the velocity of electromagnetic wave propagation and $\mathbf{a}_{k}\left(\theta_{q}\right)$ is the steering vector corresponding to $\theta_{q}$, expressed as

$$
\mathbf{a}_{k}\left(\theta_{q}\right)=\left[1, e^{-j \frac{2 \pi p_{2}}{\lambda_{k}} \sin \left(\theta_{q}\right)}, \ldots, e^{-j \frac{2 \pi p_{2} M+N-1}{\lambda_{k}} \sin \left(\theta_{q}\right)}\right]^{T}
$$

where $\lambda_{k}=c / f_{k}$ denotes the wavelength corresponding to $f_{k}$. Furthermore, $\check{\mathbf{n}}_{k}(t)$ is the additive noise vector, whose elements are assumed to be spatially and temporally white, and are independent of the target signals.

The received signal vector is converted to baseband signals corresponding to the respective frequencies, followed by

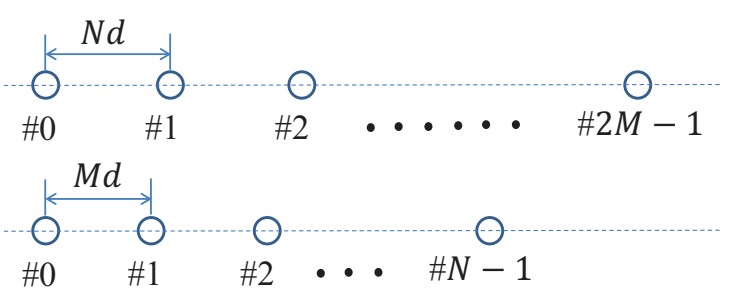

Fig. 1. The coprime array configuration.

low-pass filtering, yielding

$$
\begin{aligned}
\mathbf{x}_{k}(t) & =\sum_{q=1}^{Q} \rho_{q}(t) e^{-j \frac{4 \pi f_{k}}{c} R_{q}} \mathbf{a}_{k}\left(\theta_{q}\right)+\mathbf{n}_{k}(t) \\
& =\mathbf{A}_{k} \mathbf{B}_{k} \mathbf{d}(t)+\mathbf{n}_{k}(t),
\end{aligned}
$$

where $\mathbf{A}_{k}=\left[\mathbf{a}_{k}\left(\theta_{1}\right), \cdots, \mathbf{a}_{k}\left(\theta_{Q}\right)\right]$ and $\mathbf{d}(t)=\left[\rho_{1}(t), \cdots\right.$, $\left.\rho_{Q}(t)\right]^{T}$. In addition, the diagonal matrix

$$
\mathbf{B}_{k}=\operatorname{diag}\left(\left[e^{-j \frac{4 \pi f_{k}}{c} R_{1}}, \cdots, e^{-j \frac{4 \pi f_{k}}{c} R_{Q}}\right]\right)
$$

contains the phase information as a function of the range with respect to $f_{k}$, and $\mathbf{n}_{k}(t)$ is the noise at the filter output with a variance $\sigma_{n_{k}}^{2}$.

The cross-covariance matrix between data vectors $\mathbf{x}_{k}(t)$ and $\mathbf{x}_{l}(t)$, respectively corresponding to frequencies $f_{k}$ and $f_{l}, 1 \leq k \neq l \leq K$, is obtained as

$$
\begin{aligned}
\mathbf{R}_{\mathbf{x}_{k l}} & =\mathrm{E}\left[\mathbf{x}_{k}(t) \mathbf{x}_{l}^{H}(t)\right]=\mathbf{A}_{k} \mathbf{B}_{k} \mathbf{R}_{\mathbf{d d}} \mathbf{B}_{l}^{H} \mathbf{A}_{l}^{H} \\
& =\sum_{q=1}^{Q} \sigma_{q}^{2} e^{-j \frac{4 \pi \Delta f_{k l}}{c} R_{q}} \mathbf{a}_{k}\left(\theta_{q}\right) \mathbf{a}_{l}^{H}\left(\theta_{q}\right),
\end{aligned}
$$

where $\mathbf{R}_{\mathbf{d d}}=\mathrm{E}\left[\mathbf{d}(t) \mathbf{d}^{H}(t)\right]=\operatorname{diag}\left(\left[\sigma_{1}^{2}, \ldots, \sigma_{Q}^{2}\right]\right)$ represents the target scattering power, and $\Delta f_{k l}=f_{k}-f_{l}$ is the frequency difference. Note that we assume the target scattering coefficients to be frequency-independent for the $K \mathrm{CW}$ signals. This is satisfied by chosen close frequencies such that their maximum difference is small and is within the coherent signal bandwidth. In practice, the covariance matrix is estimated using the $T$ available samples, i.e.,

$$
\hat{\mathbf{R}}_{\mathbf{x}_{k l}}=\frac{1}{T} \sum_{t=1}^{T} \mathbf{x}_{k}(t) \mathbf{x}_{l}^{H}(t) .
$$

By vectorizing the matrix $\hat{\mathbf{R}}_{\mathbf{x}_{k l} l}$, we obtain the following measurement vector:

$$
\mathbf{z}_{k l}=\operatorname{vec}\left(\hat{\mathbf{R}}_{\mathbf{x}_{k l}}\right)=\tilde{\mathbf{A}}_{k l} \mathbf{b}_{k l},
$$

with

$$
\begin{aligned}
\tilde{\mathbf{A}}_{k l} & =\left[\tilde{\mathbf{a}}_{k l}\left(\theta_{1}\right), \ldots, \tilde{\mathbf{a}}_{k l}\left(\theta_{Q}\right)\right], \\
\mathbf{b}_{k l} & =\left[\sigma_{1}^{2} e^{-j \frac{4 \pi \Delta f_{k l}}{c} R_{1}}, \ldots, \sigma_{Q}^{2} e^{-j \frac{4 \pi \Delta f_{k l}}{c} R_{Q}}\right]^{T},
\end{aligned}
$$


where $\tilde{\mathbf{a}}_{k l}\left(\theta_{q}\right)=\mathbf{a}_{l}^{*}\left(\theta_{q}\right) \otimes \mathbf{a}_{k}\left(\theta_{q}\right)$ for $1 \leq q \leq Q$. Benefiting from the Vandermonde vector $\mathbf{a}\left(\theta_{q}\right)$, we can regard $\mathbf{z}_{k l}$ as a received signal from a single snapshot signal vector $\mathbf{b}_{k l}$ and the matrix $\tilde{\mathbf{A}}_{k l}$ corresponds to a larger virtual array which has sensors located at the cross-lags between the two subarrays. The number of DOFs is determined by the number of unique cross-lags, which is $3 M N+M-N$ [15]. As such, the increased number of DOFs enables DOA estimation of more targets than the number of sensors.

\section{TARGET LOCALIZATION USING CMT-BCS}

The signal vector $\mathbf{z}_{k l}, 1 \leq k \neq l \leq K$, in Eqn. (9) can be sparsely represented over the entire discretized angular girds as

$$
\mathbf{z}_{k l}=\boldsymbol{\Phi}_{k l} \mathbf{r}_{k l}
$$

where $\boldsymbol{\Phi}_{k l}$ is defined as the collection of steering vectors $\tilde{\mathbf{a}}_{k l}\left(\theta_{g}\right)$ over all possible grids $\theta_{g}, g=1, \ldots, G$, with $G \gg Q$. It is important to note that the DOAs $\theta_{q}, q=1, \ldots, Q$ are indicated by positions of the nonzero entries in $\mathbf{r}_{k l}$, whose values describe the corresponding coefficients $\sigma_{q}^{2} e^{-j \frac{4 \pi \Delta f_{k l}}{c} R_{q}}$. Generally, the nonzero entries corresponding to different frequency pairs share the same positions as they are associated with the same DOAs of the $Q$ targets. However, their values differ for each frequency pair. Therefore, $\mathbf{r}_{k l}$ exhibits a group sparsity across all frequency pairs and the problem described in (12) can be solve in the group sparse context.

CS techniques can be used for effective sparse reconstruction and a number of effective algorithms are available [20][24]. In this paper, we exploit the Bayesian sparse learning methods due to their superior performance and robustness to dictionary coherence. In particular, as we deal with complex entries, the CMT-BCS approach [19] is used because it avoids achieves improved estimation accuracy by utilizing group sparsity between the real and imaginary components that are merely the projection of the same complex value into two orthogonal axes.

It is clear from the above discussion that the phase term of each nonzero entry contains the range information of each individual target and is proportional to the frequency difference. A single frequency pair usually does not provide enough unambiguous range, but exploitation of multiple unequally spaced frequencies will yield desirable unambiguous range estimation [25], [26]. The detailed discussion of the range estimation is discussed in Section 3.2.

\subsection{CMT-BCS algorithm}

Assume that the entries in sparse vectors $\mathbf{r}_{k l}$ are drawn from the product of the following zero-mean Gaussian distributions:

$$
\mathbf{r}_{k l}^{g} \sim \mathcal{N}\left(\mathbf{r}_{k l}^{g} \mid \mathbf{0}, \alpha_{g} \mathbf{I}_{2}\right), g \in[1, \ldots, G],
$$

where $\mathbf{r}_{k l}^{g}$ is a vector consisting of the real part coefficient $r_{k l}^{g_{R}}$ and imagery part coefficient $r_{k l}^{g_{I}}$ with respect to the $g$ th grid in $\mathbf{r}_{k l}$. In addition, $\boldsymbol{\alpha}=\left[\alpha_{1}, \ldots, \alpha_{G}\right]^{T}$ is the variance of Gaussian pdf. It is easy to confirm that $\mathbf{r}_{k l}^{g}$ trends to be zero when $\alpha_{g}$ is set to zero [22].
To encourage the sparsity of $\mathbf{r}_{k l}$, a Gamma prior is placed on $\alpha_{g}^{-1}$, which is conjugate to the Gaussian distribution,

$$
\alpha_{g}^{-1} \sim \operatorname{Gamma}\left(\alpha_{g}^{-1} \mid a, b\right), g \in[1, \ldots, G],
$$

where $\operatorname{Gamma}\left(x^{-1} \mid a, b\right)=\Gamma(a)^{-1} b^{a} x^{-(a-1)} e^{-\frac{b}{x}}$, with $\Gamma(\cdot)$ denoting the Gamma function. It has been demonstrated in [27] that a proper choice of the hyper-parameters $a$ and $b$ encourages a sparse representation for the coefficients in $\mathbf{r}_{k l}$. As the covariance matrix is obtained on basis of received data samples in Eqn. (8), a Gaussian prior $\mathcal{N}\left(\mathbf{0}, \beta_{0} \mathbf{I}_{2}\right)$ is also placed on the additive noise. Similarly, the Gamma prior is placed on $\beta_{0}^{-1}$ with hyper-parameters $c$ and $d$.

The CMT-BCS algorithm carries out Bayesian inference by the Gibbs samplers [19]. Define $\hat{\mathbf{r}}_{k l}^{R I}=\left[\left(\hat{\mathbf{r}}_{k l}^{R}\right)^{T},\left(\hat{\mathbf{r}}_{k l}^{I}\right)^{T}\right]^{T}$ with $\hat{\mathbf{r}}_{k l}^{R}=\left[\hat{r}_{k l}^{1 R}, \ldots, \hat{r}_{k l}^{G R}\right]^{T}$ and $\hat{\mathbf{r}}_{k l}^{I}=\left[\hat{r}_{k l}^{1 I}, \ldots, \hat{r}_{k l}^{G I}\right]^{T}$. Then,

$$
\operatorname{Pr}\left(\hat{\mathbf{r}}_{k l}^{R I} \mid \mathbf{z}_{k l}, \boldsymbol{\Phi}_{k l}, \boldsymbol{\alpha}, \beta_{0}\right)=\mathcal{N}\left(\hat{\mathbf{r}}_{k l}^{R I} \mid \boldsymbol{\mu}_{k l}, \boldsymbol{\Sigma}_{k l}\right),
$$

where

$$
\begin{aligned}
& \mathbf{z}_{k l}^{R I}=\left[\operatorname{Re}\left(\mathbf{z}_{k l}\right)^{T}, \operatorname{Im}\left(\mathbf{z}_{k l}\right)^{T}\right]^{T} \\
& \boldsymbol{\mu}_{k l}=\beta_{0}^{-1} \boldsymbol{\Sigma}_{k l} \boldsymbol{\Psi}_{k l}^{T} \mathbf{z}_{k l}^{R I}, \\
& \boldsymbol{\Sigma}_{k l}=\left[\beta_{0}^{-1} \boldsymbol{\Psi}_{k l}^{T} \boldsymbol{\Psi}_{k l}+\mathbf{F}^{-1}\right]^{-1}, \\
& \boldsymbol{\Psi}_{k l}=\left[\begin{array}{cc}
\operatorname{Re}\left(\boldsymbol{\Phi}_{k l}\right) & -\operatorname{Im}\left(\boldsymbol{\Phi}_{k l}\right) \\
\operatorname{Im}\left(\boldsymbol{\Phi}_{k l}\right) & \operatorname{Re}\left(\boldsymbol{\Phi}_{k l}\right)
\end{array}\right], \\
& \mathbf{F}=\operatorname{diag}\left(\alpha_{1}, \ldots, \alpha_{G}, \alpha_{1}, \ldots, \alpha_{G}\right) .
\end{aligned}
$$

It is noted that, the real and imaginary parts share the same $\alpha$ to ensure their group sparsity. The mean and variance of each scattering coefficients can be derived, once we obtain $\alpha$ and $\beta_{0}$, which is determined by maximizing the logarithm of the marginal likelihood [19].

\subsection{Target localization}

The estimated sparse vector $\mathbf{r}_{k l}$ can be expressed as

$$
\hat{\mathbf{r}}_{k l}=\hat{\mathbf{r}}_{k l}^{R}+j \hat{\mathbf{r}}_{k l}^{I} .
$$

The DOA of the $q$ th target, $\theta_{q}$, is indicated by the position of the $q$ th nonzero entry in $\mathbf{r}_{k l}$ and the corresponding phases is obtained as

$$
\phi_{q_{k l}}=\tan ^{-1}\left(\frac{\hat{r}_{q_{k l}}^{I}}{\hat{r}_{q_{k l}}^{R}}\right),
$$

where $\hat{r}_{q_{k l}}=\hat{r}_{q_{k l}^{R}}^{R}+j \hat{r}_{q_{k l}}^{I}$ is the estimated coefficient with respect to $\theta_{q}$. Then, the corresponding range $R_{q}$ is estimated as

$$
\hat{R}_{q}=\frac{c \phi_{q_{k l}}}{4 \pi \Delta f_{k l}}
$$

without considering phase wrapping. In reality, however, phase observations are wrapped within the $[0,2 \pi)$ range. Therefore, the true phase can be expressed as

$$
\phi_{q_{k l}}^{\text {(true) }}=\phi_{q_{k l}}+2 m_{q_{k l}} \pi,
$$

where $m_{q_{k l}}$ is an unknown integer. As a result, the range estimate is subject to range ambiguity [25], i.e.,

$$
\hat{R}_{q}=\frac{c \phi_{q_{k l}}}{4 \pi \Delta f_{k l}}+\frac{c m_{q_{k l}}}{2 \Delta f_{k l}} .
$$


The latter term in Eqn. (25) implies ambiguity in range due to phase wrapping. Thus, the range can be assumed as infinite values separated by $R_{\max , k l}=c /\left(2 \Delta f_{k l}\right)$, which is referred to as the maximum unambiguous range for the frequency pair of $f_{l}$ and $f_{k}$.

As discussed earlier, a single frequency pair usually does not provide enough unambiguous range. By exploiting multiple frequencies that satisfy certain relationship, an extended unambiguous range can be achieved based on the Chinese remainder theorem [25], [26]. Without loss of generality, we denote $\tilde{k}$ th frequency pair consisting of adjacent frequencies with $\Delta f_{\tilde{k}}=f_{\tilde{k}+1}-f_{\tilde{k}}, \tilde{k}=1, \ldots, K-1$, and denote the wrapped phase observation as $\phi_{q_{\tilde{k}}}=\phi_{q_{\tilde{k}+1, \tilde{k}}}$. Then, the range estimate in Eqn. (25) can be rewritten as

$$
\hat{R}_{q}=\frac{c \phi_{q_{\tilde{k}}}}{4 \pi \Delta f_{\tilde{k}}}+\frac{c m_{q_{\tilde{k}}}}{2 \Delta f_{\tilde{k}}}=\frac{\nu_{\tilde{k}} \phi_{q_{\tilde{k}}}}{4 \pi}+\frac{m_{q_{\tilde{k}}} \nu_{\tilde{k}}}{2} .
$$

where $\nu_{\tilde{k}}=c / \Delta f_{\tilde{k}}$. By choosing $\nu_{\tilde{k}}$ as a multiple of a constant $\Delta_{d}$, which represents a range bin and its value is to be determined by the range resolution, we can express $\nu_{\tilde{k}}$ as $\nu_{\tilde{k}}=\xi_{\tilde{k}} \Delta_{d}$, where $\xi_{\tilde{k}}$ is a nonnegative integer. In this case, based on the Chinese reminder theorem, the maximum unambiguous range of the above expression, constructed from the $K-1$ frequency pairs, is

$$
R_{\max }=\frac{\Delta_{d}}{2} \cdot \operatorname{LCD}\left(\xi_{1}, \ldots, \xi_{K-1}\right),
$$

where $\operatorname{LCD}(\cdot)$ denotes the least common denominator operator. In particular, when $\xi_{1}, \ldots, \xi_{K-1}$ are mutually coprime, $\operatorname{LCD}\left(\xi_{1}, \ldots, \xi_{K-1}\right)$ takes a maximum value of $\xi_{1} \cdots \xi_{K-1}$. It is noted that, the value of $\Delta_{d}$ should be determined based on the achievable wrapped phase estimation accuracy in each frequency pair. High errors in the wrapped phase estimate may yield very high error in the resulting overall range estimate. In such a case, the robust Chinese reminder theorem allows robust range reconstruction in the presence of high wrapped phase errors [28].

\section{SIMULATION RESULTS}

In the simulations, we assume $M=3$ and $N=4$, and the unit interelement spacing is $d=\lambda_{1} / 2$, where $\lambda_{1}$ is the wavelength corresponding to $f_{1}=900 \mathrm{MHz}$. As shown in Fig. 2 , the coprime array consists of $2 M+N-1=9$ physical antennas, yielding $3 M N+M-N=35$ coarray elements. In addition, we choose $\Delta_{d}=12 \mathrm{~m}, \xi_{1}=4$, and $\xi_{2}=25$. The corresponding frequencies are respectively $f_{2}=906.25 \mathrm{MHz}$ and $f_{3}=907.25 \mathrm{MHz}$, and the resulting unambiguous range is $R_{\max }=600 \mathrm{~m}$.

We assume $Q=13$ targets, which are uniformly distributed between $-60^{\circ}$ and $60^{\circ}$ with random ranges uniformly distributed between $200 \mathrm{~m}$ and $600 \mathrm{~m}$. Identical noise power is assumed at the three frequencies, and the input SNR is $0 \mathrm{~dB}$ for all targets. As the virtual array is obtained from the estimated covariance matrix which is sensitive to the noise contamination, we use a high number of 6000 snapshots to obtain a relatively clean covariance matrix. The hyper-parameters used in the BCS algorithm are set to $a=b=c=d=0$. The maximum number of iterations in the Gibbs sampling is set to 200, and the sampler with the maximum marginal likelihood in the last 20 samples is chosen as the estimate of $\mathbf{r}_{k l}$. Fig. 3(a) shows the estimated DOA positions whereas Fig. 3(b) depicts the range estimates. For each target, the closest ranges obtained from the two frequency pairs are averaged to yield the respective range estimate. It is evident that the range positions corresponding to different frequency pairs exhibit a good agreement with the true target range for all 13 targets, whose corresponding localization results are shown in Fig. 4.

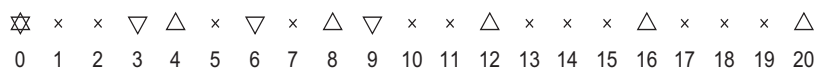

(a) Array configuration $(\triangle$ : subarray $1 ; \nabla$ : subarray 2$)$

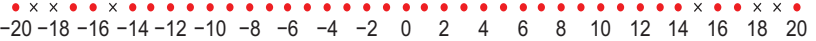
(b) Resulting coarray

Fig. 2. Array configuration and the corresponding coarray.

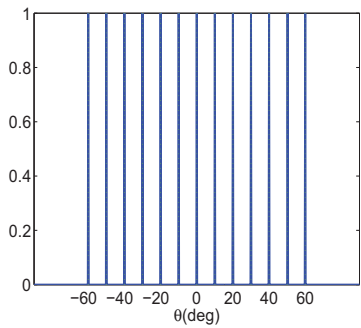

(a) DOA supports

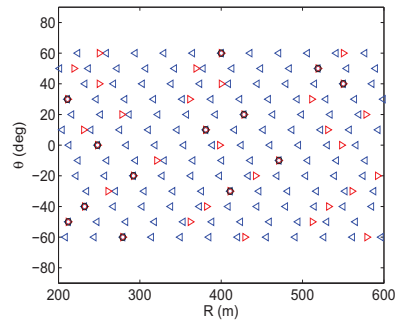

(b) Range estimates
Fig. 3. DOA supports and range estimates (०: true targets; $\triangleleft$ : estimated with $f_{1}$ and $f_{2} ; \triangleright$ : estimated with $f_{2}$ and $f_{3}$ ).

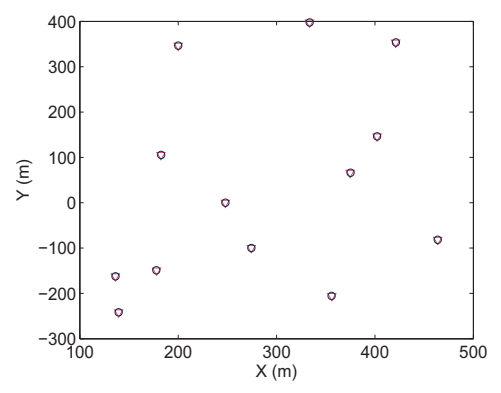

Fig. 4. Localization results ( $\circ$ : true targets; $\nabla$ : estimated).

\section{CONCLUSIONS}

In this paper, we have proposed a novel algorithm for multitarget localization using a multi-frequency coprime array. The proposed technique forms virtual arrays to achieve higher degrees-of-freedom, and properly designed multiple frequencies allow effective phase unwrapping to enable target range estimation with a large unambiguous range. As such, the proposed method localizes more targets than the number of array sensors. The effectiveness of the proposed technique was verified by simulation results. 


\section{REFERENCES}

[1] M. Skolnik, Radar Handbook, Third Edition. McGraw-Hill, 2008.

[2] D. Ribas and P. Ridao, Underwater SLAM for Structured Environments Using an Imaging Sonar. Springer, 2010.

[3] G. Mao and B. Fidan, Eds, Localization Algorithms and Strategies for Wireless Sensor Network. Information Science Reference 2009.

[4] C. Gentile and N. Alsindi, Geolocation Techniques: Principles and Applications. Springer, 2012.

[5] I. Bekkerman and J. Tabrikian, "Target detection and localization using MIMO radars and sonars," IEEE Trans. Signal Process., vol. 54, no. 10, pp. 3873-3883, Feb. 2006.

[6] Y. Zhang, M. G. Amin, and F. Ahmad, “Time-frequency analysis for the localization of multiple moving targets using dualfrequency radars," IEEE Signal Process. Lett., vol. 15, pp. 777780, 2008

[7] H. Yan, J. Li, and G. Liao, "Multitarget identification and localization using bistatic MIMO radar systems," EURASIP J. Adv. Signal Process., vol. 2008, no. ID 283483, 2008

[8] S. Qin, Y. D. Zhang, Q. Wu, and M. G. Amin, "Structureaware Bayesian compressive sensing for near-field source localization based on sensor-angle distributions," Int. J. Antennas Propagat., vol. 2015, article ID 783467, 15 pp., March 2015.

[9] Y. Zhang, X. Li, and M. G. Amin, "Principles and techniques of RFID positioning," in M. Bolic, D. Simplot-Ryl, and I. Stojmenovic (eds.), RFID Systems, Research Trends and Challenges. John Wiley, 2010.

[10] Y. Zhang, M. G. Amin, and S. Kaushik, "Localization and tracking of passive RFID tags based on direction estimation," Int. J. Antennas Propagat., vol. 2007, article ID 17426, 9 pp., Dec. 2007.

[11] B. K. Chalise, Y. D. Zhang, M. G. Amin, and B. Himed, "Target localization in a multi-static passive radar system through convex optimization," Signal Process., vol. 102, pp. 207-215, Sept. 2014.

[12] R. T. Hoctor and S. A. Kassam, "The unifying role of the coarray in aperture synthesis for coherent and incoherent imaging," Proc. IEEE, vol. 78, no. 4, pp. 735-752, April 1990.

[13] P. P. Vaidyanathan and P. Pal, "Sparse sensing with co-prime samplers and arrays," IEEE Trans. Signal Process., vol. 59, no. 2, pp. 573-586, Feb. 2011.

[14] P. Pal and P. P. Vaidyanathan, "Coprime sampling and the MUSIC algorithm," in Proc. IEEE Digital Signal Proc. Workshop and IEEE Signal Process. Education Workshop, Sedona, AZ, Jan. 2011.
[15] S. Qin, Y. D. Zhang, and M. G. Amin, "Generalized coprime array configurations for direction-of-arrival estimation," IEEE Trans. Signal Process., vol. 63, no. 6, pp. 1377-1390, March 2015.

[16] Y. D. Zhang, M. G. Amin, F. Ahmad, and B. Himed, "DOA estimation using a sparse uniform linear array with two CW signals of co-prime frequencies," in Proc. IEEE Int. Workshop on Comp. Adv. in Multi-Sensor Adaptive Process., Saint Martin, pp. 404-407, Dec. 2013.

[17] S. Qin, Y. D. Zhang, and M. G. Amin, "DOA estimation exploiting coprime frequencies," in Proc. SPIE Wireless Sensing, Localization, and Process. Conf., vol. 9103, Baltimore, MD, May 2014.

[18] D. L. Donoho, “Compressed sensing," IEEE Trans. Info. Theory, vol. 52, no. 4, pp. 1289-1306, 2006.

[19] Q. Wu, Y. D. Zhang, and M. G. Amin, "Complex multitask Bayesian compressive sensing," in Proc. IEEE ICASSP, Florence, Italy, pp. 3375-3379, May 2014.

[20] S. S. Chen, D. L. Donoho, and M. A. Saunders, "Atomic decomposition by basis pursuit," SIAM J. Scientific Computing, vol. 20, no. 1, pp. 33-61, 1998.

[21] J. A. Tropp and A. C. Gilbert, "Signal recovery from random measurements via orthogonal matching pursuit," IEEE Trans. Info. Theory, vol. 53, no. 12, pp. 4655-4666, 2007.

[22] S. Ji, Y. Xue, and L. Carin, "Bayesian compressive sensing," IEEE Trans. Signal Process., vol. 56, no. 6, pp. 2346-2356, Aug. 2008.

[23] S. Ji, D. Dunson, and L. Carin, "Multitask compressive sensing," IEEE Trans. Signal Process., vol. 57, no. 1, pp. 92-106, 2009.

[24] Q. Wu, Y. D. Zhang, M. G. Amin, and B. Himed, "Multitask Bayesian compressive sensing exploiting intra-task correlation," IEEE Signal Process. Lett., vol. 22, no. 4, pp. 430-434, 2015.

[25] X.-G. Xia and G. Wang, "Phase unwrapping and a robust Chinese remainder theorem," IEEE Signal Process. Lett., vol. 14, no. 4, pp. 247-250, 2007.

[26] X. Li, Y. D. Zhang, and M. G. Amin, "Multifrequency-based range estimation of RFID tags," in Proc. IEEE RFID Conf., pp. 147-154, Apr. 2009.

[27] M. E. Tipping, "Sparse Bayesian shrinkage and selection learning and the relevance vector machine," J. Machine Learning Research, vol. 1, no. 9, pp. 211-244, 2001.

[28] X. Li, H. Liang, and X.-G. Xia, "A robust Chinese remainder theorem with its applications in frequency estimation from undersampled waveforms," IEEE Trans. Signal Process., vol. 57, no. 11, pp. 4314-4322, 2009. 